\title{
Partially Twisted Boundary Conditions in Lattice Simulations*
}

\author{
Jonathan Flynn, Andreas Jüttner, Christopher Sachrajda ${ }^{\dagger}$ \\ School of Physics and Astronomy, University of Southampton, \\ Highfield, Southampton, SO17 1BJ, United Kingdom \\ E-mail: \{jflynn, juettner, cts\} @phys.soton.ac.uk
}

\section{Giovanni Villadoro}

Dip. di Fisica, Università di Roma "La Sapienza" and INFN-Sezione di Roma Piazzale A. Moro 2, I-00185 Rome, Italy

E-mail: giovanni.villadoro@romal.infn.it

\begin{abstract}
We use chiral perturbation theory to investigate twisted and partially twisted boundary conditions which allow access to momenta other than integer multiples of $2 \pi / L$ on a lattice with spatial volume $L^{3}$. For $K \rightarrow \pi \pi$ decays we show that the breaking of isospin symmetry by the twisted boundary conditions implies that the amplitudes cannot be determined in general. We find numerical evidence for the result that the finite volume effects of the boundary conditions are exponentially suppressed for quantities without final state interactions (meson masses and meson-to-vacuum matrix elements) in a simulation with partial twisting on a sea of $N_{f}=2$ non-perturbatively improved Wilson quarks.
\end{abstract}

XXIIIrd International Symposium on Lattice Field Theory

25-30 July 2005

Trinity College, Dublin, Ireland

\footnotetext{
*SHEP-0530

† Speaker.
} 


\section{Introduction}

In lattice simulations of QCD on a cubic volume $\left(V=L^{3}\right)$ with periodic boundary conditions imposed on the fields, hadronic momenta, $\vec{p}$, are quantized according to $p_{i}=2 \pi / L \times n_{i}$, where $i=1,2,3$ and the $n_{i}$ are integers. This implies that on currently available lattices the gaps between neighbouring momenta are large, typically about $500 \mathrm{MeV}$ or so, limiting the phenomenological reach of the simulations. In ref. $[1,2]{ }^{1}$ Bedaque proposed the use of twisted boundary conditions on the quark fields $(q(x))$, e.g.

$$
q\left(x_{i}+L\right)=e^{i \theta_{i}} q\left(x_{i}\right)
$$

so that the momentum spectrum is

$$
p_{i}=n_{i} \frac{2 \pi}{L}+\frac{\theta_{i}}{L}
$$

Thus by varying the twisting angles, the $\theta_{i}$ 's, arbitrary momenta can be reached. In refs. [3, 4] we have investigated the use of twisted boundary conditions theoretically and numerically and in this note we briefly report the conclusions of these studies.

\section{Finite-Volume Effects with Twisted Boundary Conditions}

The three main results from ref. [3] are:

1. For physical quantities without final state interactions, such as masses or matrix elements of local operators between states consisting of the vacuum or a single hadron, the flavour symmetry breaking induced by the twist only affects the finite-volume corrections, which nevertheless remain exponentially small.

2. For physical quantities without final state interactions, one can also use partially twisted boundary conditions in which the sea quarks satisfy periodic boundary conditions, but some or all of the valence quarks satisfy twisted boundary conditions, with exponential precision in the volume. This implies that in unquenched simulations it is not necessary to generate new gluon configurations for every choice of twisting angle, making the method much more practicable.

3. For amplitudes which do involve final state interactions (such as $K \rightarrow \pi \pi$ decays), it is not possible in general to extract the physical matrix elements using twisted boundary conditions (at least without introducing new ideas)

We now briefly discuss each of these results in turn.

\subsection{Result 1 .}

The choice of boundary conditions for the fields only affects the finite-volume corrections and when extracting physical quantities we need to establish that these corrections are negligibly small. Infrared effects dominated by the pseudo-Goldstone bosons can be evaluated using Chiral Perturbation Theory and in ref. [3] we derive the chiral Lagrangian corresponding to the twisted boundary conditions. Consider $S U(N)_{L} \times S U(N)_{R}$ chiral symmetry and impose the twisted boundary conditions on the quark fields $q\left(x_{i}+L\right)=U_{i} q\left(x_{i}\right)=\exp \left(i \theta_{i}^{a} T^{a}\right) q\left(x_{i}\right)$, where we take the $U_{i}$

\footnotetext{
${ }^{1}$ See also the references cited in $[1,2]$ for earlier related ideas.
} 
to be diagonal $N \times N$ unitary matrices and the $T^{a}$ 's are the generators of the Cartan subalgebra of the (light) flavour $U(N)_{V}$ group. In the chiral Lagrangian these boundary conditions imply that $\Sigma(x)$, the coset representative of $S U(N)_{L} \times S U(N)_{R} / S U(N)_{V}$ satisfies the boundary conditions $\Sigma\left(x_{i}+L\right)=U_{i} \Sigma\left(x_{i}\right) U_{i}^{\dagger}$. The Feynman rules for the Chiral Lagrangian are the usual ones, except that the momentum spectrum for the Pseudo-Goldstone bosons is that expected of a particle composed of a quark-antiquark pair, each with a momentum given in terms of its twist by eq. (1.2). The effect of the twist is a change in the charged meson's dispersion relation,

$$
E_{\pi, \rho}^{2}=m_{\pi, \rho}^{2}+\left(\frac{2 \pi \vec{n}-\vec{\theta}}{L}\right)^{2}
$$

where $m_{\pi / \rho}$ is the meson mass and $\theta_{i}=\theta_{u, i}-\theta_{d, i}$, the difference of the twists of the $u$ and $d$ quarks.

As an example consider the finite-volume corrections in $f_{\pi}$, the leptonic decay constant of the $\pi$-mesons. As $L \rightarrow \infty$ the leading corrections are found to be:

$\frac{\Delta f_{\pi^{ \pm}}}{f_{\pi^{ \pm}}} \rightarrow-3 \frac{m_{\pi}^{2}}{f_{\pi}^{2}} \frac{e^{-m_{\pi} L}}{\left(2 \pi m_{\pi} L\right)^{3 / 2}}\left(\frac{1}{3} \sum_{i=1}^{3} \cos \theta_{i}+1\right), \frac{\Delta f_{\pi^{0}}}{f_{\pi^{0}}} \rightarrow-3 \frac{m_{\pi}^{2}}{f_{\pi}^{2}} \frac{e^{-m_{\pi} L}}{\left(2 \pi m_{\pi} L\right)^{3 / 2}}\left(\frac{2}{3} \sum_{i=1}^{3} \cos \theta_{i}\right)$

where $\Delta(X) / X \equiv(X(L)-X(\infty)) / X(\infty)$. The results in eq.(2.2) illustrate the point that whereas the finite-volume corrections do depend on the boundary conditions as expected, they nevertheless remain exponentially small (see ref. [3] for further examples). The difference of the correction terms in eq.(2.2) is a manifestation of the violation of isospin by the boundary conditions.

\subsection{Result 2.}

The derivation of the Chiral Lagrangian and the corresponding Feynman rules with Partially Twisted boundary conditions follows similar steps to that for partially quenched QCD. As an illustration of the results consider the finite-volume corrections to $f_{K}$, the leptonic decay constant of the $K$-meson, with the $d$ and $s$ quarks satisfying periodic boundary conditions and the $u$ quark with (a) untwisted, (b) fully twisted (c) partially twisted boundary conditions:

$$
\frac{\Delta f_{K^{ \pm}}}{f_{K^{ \pm}}} \rightarrow\left\{\begin{array}{l}
-\frac{9}{4} \frac{m_{\pi}^{2}}{f_{\pi}^{2}} \frac{e^{-m_{\pi} L}}{\left(2 \pi m_{\pi} L\right)^{3 / 2}} \\
-\frac{m_{\pi}^{2}}{f_{\pi}^{2}} \frac{e^{-m_{\pi} L}}{\left(2 \pi m_{\pi} L\right)^{3 / 2}}\left(\frac{1}{2} \sum_{i=1}^{3} \cos \theta_{i}+\frac{3}{4}\right) \\
-\frac{m_{\pi}^{2}}{f_{\pi}^{2}} \frac{e^{-m_{\pi} L}}{\left(2 \pi m_{\pi} L\right)^{3 / 2}}\left(\sum_{i=1}^{3} \cos \theta_{i}-\frac{3}{4}\right)
\end{array}\right.
$$

This example illustrates again that, in general, the finite-volume corrections are different for the three cases but they are always exponentially small.

\subsection{Result 3 .}

To illustrate the problem in using twisted boundary conditions for processes with final state interactions consider $K \rightarrow \pi \pi$ decays in the $I=0$ channel. The twisted boundary conditions break isospin symmetry which implies that energy eigenstates are no longer states with definite isospin. 
This is manifest even in the free theory. As an example consider the two pions to be in the centre-ofmass frame and let the $u$ and $d$ quark satisfy twisted and periodic boundary conditions respectively so that the lowest momenta of the neutral and charged pions are $\vec{p}_{\pi^{0}}=\overrightarrow{0}$ and $\vec{p}_{\pi^{ \pm}}= \pm \vec{\theta} / L$. The corresponding energies of the two-pion states are then $E_{\pi^{0} \pi^{0}}=2 m_{\pi}$ and $E_{\pi^{+} \pi^{-}}=2 \sqrt{m_{\pi}^{2}+\theta^{2} / L^{2}}$ making it explicit that the energy and isospin eigenstates are different.

The interacting theory contains $\pi^{+} \pi^{-} \leftrightarrow \pi^{0} \pi^{0}$ transitions which complicate the analysis very significantly making it impossible to relate the finite-volume energy eigenstates to the infinitevolume energy and isospin eigenstates $|\pi \pi\rangle_{I=0}$ and $|\pi \pi\rangle_{I=2}$. Without new ideas, it not therefore not possible to determine physical $I=0 K \rightarrow \pi \pi$ amplitudes using twisted boundary conditions with finite-volume corrections under control.

There is a further difficulty which we would like to exhibit. Even if we were able to overcome or circumvent the problem above (for example, by using G-parity boundary conditions proposed by Christ and $\operatorname{Kim}[5]^{2}$ ). The finite-volume effects which decrease only slowly, i.e. as powers of the volume, come from the propagation of two-pion states illustrated by the diagram:

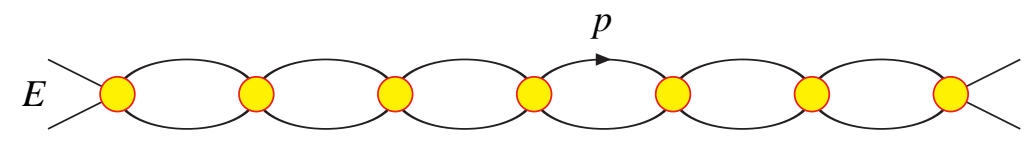

The circles represent insertions which are two-particle irreducible in the s-channel. In evaluating finite-volume corrections we replace the sums over the discrete momenta in a finite volume by integrals over continuous infinite-volume momenta using the Poisson summation formula:

$$
\frac{1}{L^{3}} \sum_{\vec{p}} f(\vec{p})=\sum_{\vec{l} \in Z^{3}} \int \frac{d^{3} p}{(2 \pi)^{3}} e^{i L \vec{l} \cdot \vec{p}} f(\vec{p}) .
$$

For two-particles propagating with an energy above the two-pion threshold $\left(E>2 m_{\pi}\right)$ there are poles in $f(\vec{p})$ (corresponding to the cut in infinite volume) and this implies that the terms with $l \neq 0$ in eq. (2.4) are not exponentially small. For $I=0 K \rightarrow \pi \pi$ decays, the sea quarks contribute to the final-state interactions as illustrated for example, by the following diagram:

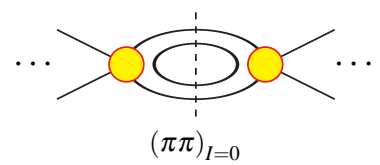

Therefore, unless the same boundary conditions are imposed for both the valence and the sea quarks, there is a breakdown of unitarity induced by the partially twisted boundary conditions and the finite-volume corrections are not under control. This is analogous to the inconsistencies encountered in quenched and partially quenched QCD for $K \rightarrow \pi \pi$ decays above the two-pion threshold in refs. [6,7].

\section{Numerical study of partially twisted boundary conditions}

This section describes the results of an exploratory numerical study of partially twisted boundary conditions, in which we investigate, whether the meson's momentum follows eq.(2.1) and

\footnotetext{
${ }^{2}$ The twist angle is now fixed to be $\pi$ and so the benefit of using partial twisting are not so clear.
} 
whether the meson decay constants $f_{\pi}$ and $f_{\rho}$ and the matrix element $Z_{P} \equiv\langle 0|P| \pi\rangle$ of the pseudo scalar density $P$ are independent of the twisting angle.

The study has been carried out on 200 independent, non-perturbatively improved $N_{f}=2$ Wilson gauge configurations with the following specifications: $\beta=5.2,(L / a)^{3} \times T / a=16^{3} \times 32$, $a \approx 0.1 \mathrm{fm}, \kappa_{\text {val }}=\kappa_{\text {sea }}=0.13500$ and 0.13550 corresponding to $m_{\pi} / m_{\rho}=0.697(11)$ and $0.566(16)$ (see also $[8,9]$ ).

We evaluated mesonic correlation functions (details in ref. [4]) for all possible pairs of valence quark twisting angles $\vec{\theta}_{u, d} \in\{\overrightarrow{0},(2,0,0),(0, \pi, 0)$ and $(3,3,3)\}$ at the Fourier momenta $\vec{p}_{\text {lat }}=\overrightarrow{0}$ and $(0, \pm 2 \pi / L, 0)$. In addition we also evaluated all quantities at $\left|\vec{p}_{\text {lat }}\right|=\sqrt{2} \times 2 \pi / L$ and $\sqrt{3} \times 2 \pi / L$ for vanishing twist. The jack-knife analysis of the resulting correlation functions focused on the momentum dependence of $E_{\pi}$ and $E_{\rho}$ and we also monitored the un-improved bare decay constants $f_{\pi}$ and $f_{\rho}$ and matrix element $Z_{P}$ under the variation of the momentum.

Here we discuss only the data for the set at $m_{\pi} / m_{\rho}=0.697(11)$ and refer the reader to ref. [4] for details and qualitatively equivalent results for the lighter data set. The plots in figure 1 show our results as a function of $(\vec{p} L)^{2}$. The positions of the discrete Fourier momenta $\left|\vec{p}_{\text {lat }} L\right|=0$, $2 \pi, \sqrt{2} \times 2 \pi$ and $\sqrt{3} \times 2 \pi$ which can be reached without twisting are indicated by dashed vertical lines. In each plot the (blue) triangles correspond to points in which the correlation function was evaluated with $\vec{p}_{\text {lat }}=\overrightarrow{0}$, but with all possible pairs of $\vec{\theta}_{u}$ and $\vec{\theta}_{d}$. The (red) diamonds and (green) squares represent the results obtained with $\vec{p}_{\text {lat }}=(0,2 \pi / L, 0)$ and $\vec{p}_{\text {lat }}=-(0,2 \pi / L, 0)$ respectively, combined with all possible pairs of $\vec{\theta}_{u}$ and $\vec{\theta}_{d}$. The four points with $\vec{\theta}_{u}=\vec{\theta}_{d}=\overrightarrow{0}$ but $\left|\vec{p}_{\text {lat }}\right|=$ $0,2 \pi / L, \sqrt{2} \times 2 \pi / L$ and $\sqrt{3} \times 2 \pi / L$ are denoted by (black) circles. The three main results are:

1. The energies of $\pi$ and $\rho$-mesons (with masses below the two-pion threshold) satisfy eq. (2.1) very well, particularly for small values of the momentum where lattice artifacts are small.

2. The values of the leptonic decay constants $f_{\pi}$ and $f_{\rho}$ and of the matrix element $Z_{P}$ are independent of the twisting angles for small values of the momentum. Deviations for large momenta are of the same magnitude when using twisted boundary conditions or only Fourier transformation to induce momentum.

3. Twisted boundary conditions do not introduce additional noise in the data. The combined statistical and systematic error on the meson masses and matrix elements increases smoothly when increasing the meson's momentum by varying the angles $\vec{\theta}_{u, d}$. However, when comparing results obtained with twisted and periodic boundary conditions with similar momenta (i.e. around $2 \pi / L$ or $\sqrt{2}(2 \pi / L)$ ), the errors are found to be comparable.

\section{Conclusions}

The theoretical and numerical results presented in this note demonstrate that the use of partially twisted boundary conditions does indeed improve the momentum resolution in lattice phenomenology at relatively little cost for physical quantities without final-state interactions. We now look forward to applying these techniques to studies of weak matrix elements and hadronic structure. We have also demonstrated that twisted and partially twisted boundary conditions cannot be applied in general to physical quantities with final state interactions such as $K \rightarrow \pi \pi$ decays with isospin 0 . 

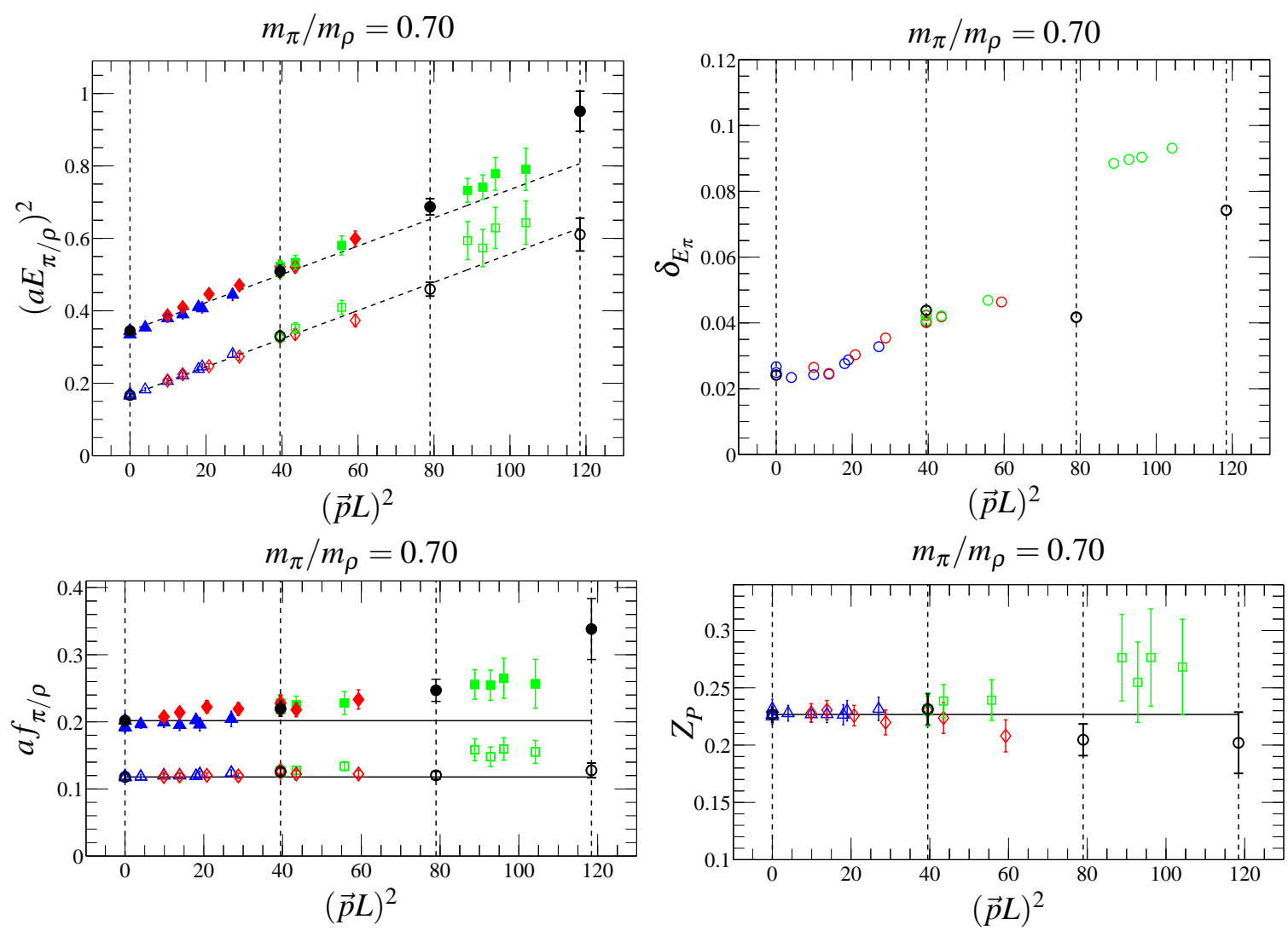

Figure 1: Results for the case $m_{\pi} / m_{\rho}=0.70$. The plots in the first line illustrate the results for the dispersion relation for the $\pi$ and the $\rho$ (empty and full symbols respectively) and the associated error in the case of the $\pi$ as a function of the momentum. The second line shows the results for the $\pi$ and $\rho$ decay constant and $Z_{P}$. In each plot the horizontal lines represent the central value at $\vec{p}_{\text {lat }}=\vec{\theta}_{1}=\vec{\theta}_{2}=0$. Note also in all the four plots the superimposed data points at $\left|\vec{p}_{\text {lat }}\right|=0,2 \pi / L$, which correspond to the cases $\vec{\theta}_{u}-\vec{\theta}_{d}=0$.

\section{References}

[1] P. F. Bedaque, Phys. Lett. B 593 (2004) 82 [arXiv:nucl-th/0402051].

[2] G. M. de Divitiis, R. Petronzio and N. Tantalo, Phys. Lett. B 595 (2004) 408 [arXiv:nucl-th/0405002].

[3] C. T. Sachrajda and G. Villadoro, Phys. Lett. B 609 (2005) 73 [arXiv:hep-lat/0411033].

[4] J. M. Flynn, A. Jüttner and C. T. Sachrajda [UKQCD Collaboration], arXiv:hep-lat/0506016.

[5] C. h. Kim and N. H. Christ, Nucl. Phys. Proc. Suppl. 119 (2003) 365 [arXiv:hep-lat/0210003].

[6] C. J. D. Lin, G. Martinelli, E. Pallante, C. T. Sachrajda and G. Villadoro, Phys. Lett. B 553 (2003) 229 [arXiv:hep-lat/0211043].

[7] C. J. D. Lin, G. Martinelli, E. Pallante, C. T. Sachrajda and G. Villadoro, Phys. Lett. B 581 (2004) 207 [arXiv:hep-lat/0308014].

[8] C.R. Allton, et al., Phys. Rev. D 65 (2002) 054502 [arXiv:hep-lat/0107021].

[9] C.R. Allton, et al., Phys. Rev. D 70 (2004) 014501 [arXiv:hep-lat/0403007]. 